\title{
利用燃烧反应快速升温方法制备 $\mathrm{Al}_{2} \mathrm{O}_{3} / \mathrm{YAG}$ 共晶陶瓷
}

\author{
李涁 ${ }^{(1) 2}$ ，项铭禹 ${ }^{(1)}$ ，包晗 ${ }^{(1)}$ ，张金咏 ${ }^{(1)}$ ，王为民 ${ }^{(1)}$ ，傅正义 ${ }^{(1 *}$ \\ (1) 武汉理工大学材料复合新技术国家重点实验室, 武汉 430070; \\ (2) School of Natural Science, Sun Moon University, Chungnam 336-708, South Korea \\ *联系人, E-mail: zyfu@whut.edu.cn \\ 2014-05-13 收稿, 2014-09-28 接受 \\ 国家自然科学基金(51161140399)和科技部国际合作项目(S2010GR0771)资助
}

\begin{abstract}
摘要 $\mathrm{Al}_{2} \mathrm{O}_{3} / \mathrm{Y}_{3} \mathrm{Al}_{5} \mathrm{O}_{12}(\mathrm{YAG})$ 共晶陶瓷具有优异的热力学稳定性, 被认为是超高温强氧化性环境 下长期工作的候选材料之一。 已报道的共晶陶瓷制备方法均依赖复杂的高温设备. 本文采用了 一种新的燃烧反应快速升温方法熔化和制备共晶陶瓷, 具有升温速度快(可达 $\left.2000^{\circ} \mathrm{C} / \mathrm{min}\right)$ 、温度 高、冷却速率快、设备简单等特点. 研究了利用该方法制备的 $\mathrm{Al}_{2} \mathrm{O}_{3} / \mathrm{YAG}$ 共晶陶瓷的微观结构和 性能. 结果表明制备的共晶陶瓷含有 $\mathrm{Al}_{2} \mathrm{O}_{3}$ 和 $\mathrm{YAG}$ 相, 两相耦合生长, 相界面匹配良好, 没有杂 相存在; 微观组织除具有典型的“象形文字(CS, Chinese script)” 结构外, 还得到特殊的形貌, 并分 析了微观结构形成的机理. $\mathrm{Al}_{2} \mathrm{O}_{3} / \mathrm{YAG}$ 共晶陶瓷硬度为 $20.52 \mathrm{GPa}$, 断裂韧性为 $2.64 \mathrm{MPa} \mathrm{m}^{1 / 2}$, 裂 纹以穿晶断裂方式扩展. 共晶陶瓷经过 $1500^{\circ} \mathrm{C}$ 处理 $20 \mathrm{~h}$ 后, 样品无失重、也无明显结构变化, 具 有较好的高温结构稳定性.
\end{abstract}

关键词

燃烧反应

快速升温

共晶陶瓷

$\mathrm{Al}_{2} \mathrm{O}_{3} / \mathrm{YAG}$

共晶结构
氧化铝基共晶陶瓷能够在接近熔点的高温氧化 环境下保持良好的力学性能与结构稳定性, 是一类 重要的高温结构材料, 有望替代镍基高温合金制造 航空发动机涡轮叶片 ${ }^{[1 ~ 4]}$. 目前报道的氧化铝基共晶

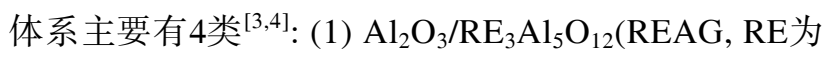
稀土元素，下同); (2) $\mathrm{Al}_{2} \mathrm{O}_{3} / \mathrm{REAlO}_{3}(\mathrm{REAP}) ;$ $\mathrm{Al}_{2} \mathrm{O}_{3} / \mathrm{ZrO}_{2}$; (4) $\mathrm{Al}_{2} \mathrm{O}_{3} / \mathrm{REAG} / \mathrm{ZrO}_{2}$. 其中 $\mathrm{Al}_{2} \mathrm{O}_{3} / \mathrm{YAG}$ 体系具有不规则网络状共晶结构, 在高温下仍具有 接近室温的力学性能, 是典型的氧化铝基二元共晶 体系, 得到了最广泛的研究 ${ }^{[5 \sim 8]}$. 近年来, 对氧化铝 基共晶陶瓷的报道主要集中于新型快速制备技术、原 料组分和凝固条件对共晶结构与力学性能的影响等 几方面. 苏海军等人 ${ }^{[9 \sim 11]}$ 利用激光定向凝固技术制备 了致密的 $\mathrm{Al}_{2} \mathrm{O}_{3} / \mathrm{YAG}, \mathrm{Al}_{2} \mathrm{O}_{3} / \mathrm{YAG} / \mathrm{ZrO}_{2}$ 等共晶陶瓷并 进行了研究; $\mathrm{Fu}$ 等人 ${ }^{[12]}$ 研究了 $\mathrm{Al}_{2} \mathrm{O}_{3} / \mathrm{YAG} / \mathrm{ZrO}_{2}$ 共晶 体系熔体从不同温度冷却与得到的共晶形貌的关系; Zhao等人 ${ }^{[13]}$ 和 Liu等人 ${ }^{[14]}$ 分别以超重力场辅助燃烧
合成技术进行了共晶陶瓷的制备.

目前报道的氧化铝基共晶陶瓷制备技术对设备 要求普遍较高. 本文采用燃烧反应作为热源来实现 $\mathrm{Al}_{2} \mathrm{O}_{3} / \mathrm{YAG}$ 共晶陶瓷的快速制备，具有设备简单、周 期短、能耗低等优点, 是一种共晶陶瓷快速制备新技 术. 一些特定的反应物之间能够发生剧烈的燃烧反 应, 燃烧反应速度快而且燃烧温度高. 根据燃烧体系 的选取, 其升温速率可超过 $2000^{\circ} \mathrm{C} / \mathrm{min}$, 反应温度可 以超过 $3000^{\circ} \mathrm{C}$, 数分钟内反应即可结束并降至室温. 利用燃烧反应的制备技术已经在纳米晶陶瓷的快速 致密化方面展现出很大潜力 ${ }^{[15,16]}$. 利用 $\mathrm{Ti}$ 与 $\mathrm{C}$ 的燃烧 反应将共晶组分的原料熔化, 并随反应冷却凝固, 同 时对所制备材料的物相组成、显微形貌和部分力学性 能进行了研究与探讨.

\section{1 实验}

共晶陶瓷原料为高纯 $\alpha-\mathrm{Al}_{2} \mathrm{O}_{3}$ 粉体 (99.99\%) 和

引用格式: 李彬, 项铭禹, 包晗, 等. 利用燃烧反应快速升温方法制备 $\mathrm{Al}_{2} \mathrm{O}_{3} / \mathrm{YAG}$ 共晶陶瓷. 科学通报, 2015, 60: 322-327

$\mathrm{Li} \mathrm{B}$, Xiang M Y, Bao H, et al. Fabrication of $\mathrm{Al}_{2} \mathrm{O}_{3} / \mathrm{YAG}$ eutectic ceramics utilizing a fast combustion reaction heating method (in Chinese). Chin Sci Bull, 2015, 60: 322-327, doi: 10.1360/N972014-00450 
$\mathrm{Y}_{2} \mathrm{O}_{3}$ 粉体 (99.99\%). 依据图 1所示的 $\mathrm{Al}_{2} \mathrm{O}_{3}-\mathrm{Y}_{2} \mathrm{O}_{3}$ 平衡 相图, 按摩尔比 $81.6: 18.4$ 的共晶组成进行配料, 将混 合粉体以乙醇为研磨介质混合 $12 \mathrm{~h}$, 旋转蒸发干燥后 以 $8 \mathrm{MPa}$ 压制成直径 $10 \mathrm{~mm}$ 的圆片, 之后在冷等静压 机中以200 MPa进行二次压制, 处理后的坏体装人石 墨坩埚中.

利用 $\mathrm{Ti}$ 和 $\mathrm{C}$ 的燃烧反应 $(\mathrm{Ti}+\mathrm{C} \rightarrow \mathrm{TiC})$ 产生的热量 熔化 $\mathrm{Al}_{2} \mathrm{O}_{3}$ 与 $\mathrm{Y}_{2} \mathrm{O}_{3}$ 的混合粉体. 燃烧反应用 $\mathrm{Ti}$ 粉 (99.5\%)和碳黑(99\%)以摩尔比 $1: 1$ 进行配料, 混合 $12 \mathrm{~h}$, 压制成直径 $50 \mathrm{~mm}$, 厚度 $35 \mathrm{~mm}$ 的坯体, 压制时将装 有共晶陶瓷生坏的石墨坩埚置于坏体中间位置, 为避 免石墨坩埚被压坏, 压力控制在 $10 \mathrm{MPa}$ 以下. 燃烧反 应装置如图 2 所示 ${ }^{[15]}$, 反应坯体装在 1 个钢制模具中, 用干燥的沙子填充坏体与模具之间的空隙, 实验开始 时向铇丝线圈通人 $80 \mathrm{~A}$ 的大电流, 产生的电阻热瞬间 引发燃烧反应. 反应过程中的温度由WRe5/26型热电 偶测量, 测得的时间-温度曲线如图3所示.

采用日本理学D/Max-RB型X射线衍射仪(XRD) 分析共晶陶瓷的晶相组成, 日本日立S3200N型扫描 电子显微镜 (SEM) 观察材料的微观形貌并通过其自 带能谱仪(EDS)进行微区半定量元素分析, 日本电子

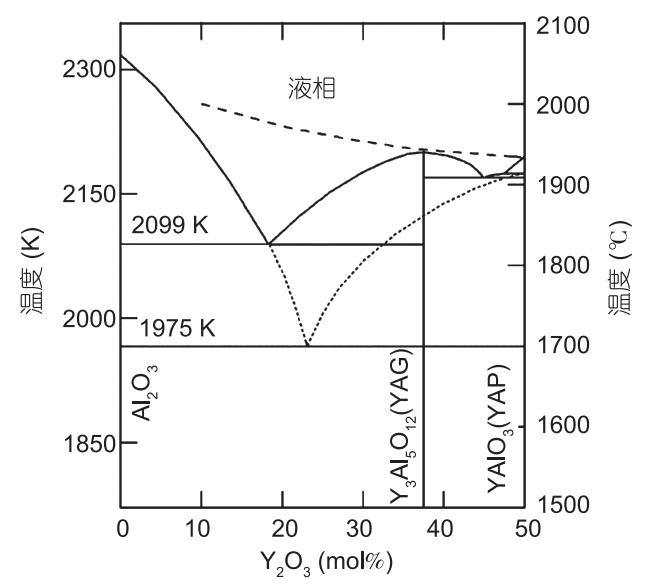

图 $1 \mathrm{Al}_{2} \mathrm{O}_{3}-\mathrm{Y}_{2} \mathrm{O}_{3}$ 体系相图

Figure 1 Phase diagram of $\mathrm{Al}_{2} \mathrm{O}_{3}-\mathrm{Y}_{2} \mathrm{O}_{3}$

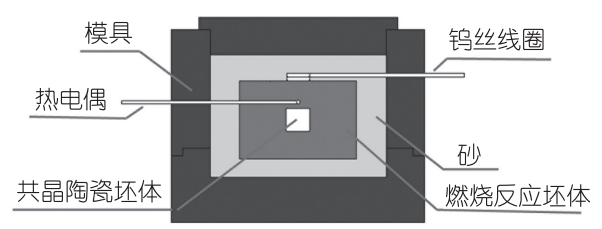

图 2 燃烧反应装置装配示意图

Figure 2 Schematic representation of experiment process

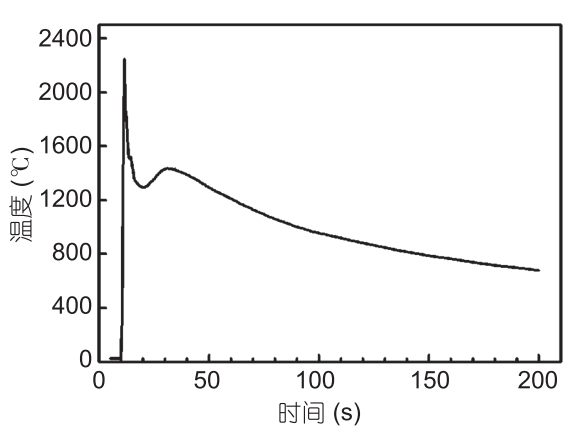

图 3 燃烧反应时间-温度关系曲线

Figure 3 The temperature curve depending on time

JEM-2010型高分辨透射电子显微镜 (HRTEM) 观察 $\mathrm{Al}_{2} \mathrm{O}_{3}$ 与 $\mathrm{YAG}$ 的相界面并通过其自带EDS进行微区半 定量元素分析. 采用美国Wolpert 430SVD型维氏硬 度计测定材料的维氏硬度并根据式(1)计算断裂韧性 $K_{\text {IC }}{ }^{[17,18]}$.

$$
K_{\mathrm{IC}}=0.0154\left(E / H_{\mathrm{V}}\right)^{1 / 2} P / C^{3 / 2},
$$

其中, $E$ 为材料的杨氏模量, $H_{\mathrm{V}}$ 为材料的维氏硬度, $P$ 为测量硬度时的载荷, $C$ 为裂纹长度, 取 $\mathrm{Al}_{2} \mathrm{O}_{3} / \mathrm{YAG}$ 共晶陶瓷的理论值 $345.2 \mathrm{GPa}^{[9]}$.

\section{2 结果与讨论}

燃烧反应开始后燃烧波由上向下快速推进, 将 坩埚中的共晶陶瓷坏体加热熔融, 并在30 s内达到最 高温度后开始自然冷却(图3), 坩埚中的熔体随温度 下降凝固, 得到共晶陶瓷材料, 整个过程在数分钟之 内完成. 所得共晶陶瓷的表面由于熔体表面张力作 用而有一定曲率，未发现与石墨坩埚黏附.

图4为垂直于燃烧波传播方向上 $\mathrm{Al}_{2} \mathrm{O}_{3} / \mathrm{YAG}$ 共晶 陶瓷横截面的XRD图谱. 结果显示, 图谱中只有 $\mathrm{Al}_{2} \mathrm{O}_{3}$ 和 $\mathrm{YAG}$ 两相, $\mathrm{Al}_{2} \mathrm{O}_{3}(116)$ 晶面和 $\mathrm{YAG}(420)$ 晶面 存在明显取向, 表明在凝固过程中该方向上 $\mathrm{Al}_{2} \mathrm{O}_{3}(116)$ 晶面和YAG(420)晶面上发生了择优生长.

图 5(a)为 $\mathrm{Al}_{2} \mathrm{O}_{3} / \mathrm{YAG}$ 共晶陶瓷抛光表面显微形 貌. 结合EDS分析可知颜色较深相为 $\mathrm{Al}_{2} \mathrm{O}_{3}$, 较浅相 为 $Y A G$, 两相相互交织、紧密相连. $\mathrm{Al}_{2} \mathrm{O}_{3}$ 和 $\mathrm{YAG}$ 的熔 化熵 $\Delta S_{\mathrm{f}}$ 分别为 $5.74 R(48 \mathrm{~J} / \mathrm{mol})$ 和 $14.72 R(122 \mathrm{~J} / \mathrm{mol}), R$ 为气体常数. 根据Jackson-Hunt理论 ${ }^{[19]}, \Delta S_{\mathrm{f}}>5 R$ 的晶 体倾向于小平面生长, $\mathrm{Al}_{2} \mathrm{O}_{3} / \mathrm{YAG}$ 共晶陶瓷是典型的 小平面-小平面生长的非规则共晶, 需要更大的动力 学生长过冷度 ${ }^{[20]}$. 燃烧反应结束后, $\mathrm{Al}_{2} \mathrm{O}_{3} / \mathrm{Y}_{2} \mathrm{O}_{3}$ 熔体 随反应坏体在室温下快速冷却, 产生较大动力学生 


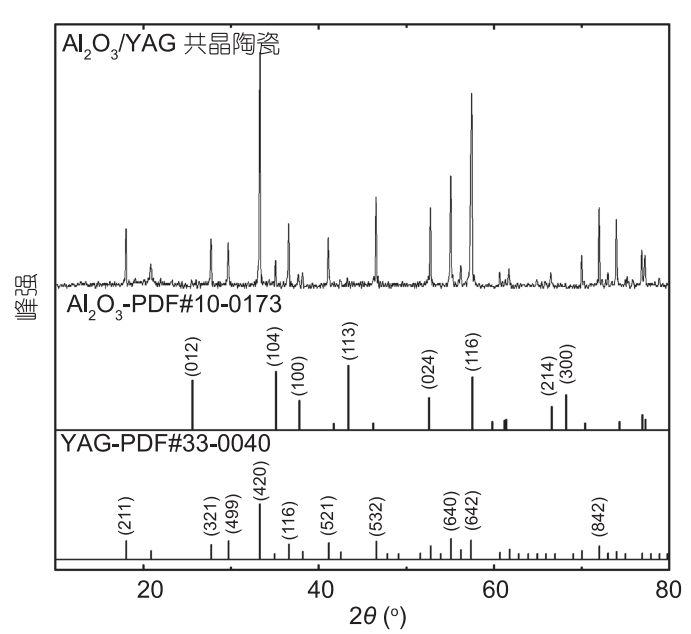

图 $4 \mathrm{Al}_{2} \mathrm{O}_{3} / \mathrm{YAG}$ 共晶陶瓷横截面的 XRD 图谱

Figure 4 XRD pattern of the $\mathrm{Al}_{2} \mathrm{O}_{3} / \mathrm{YAG}$ eutectic ceramics
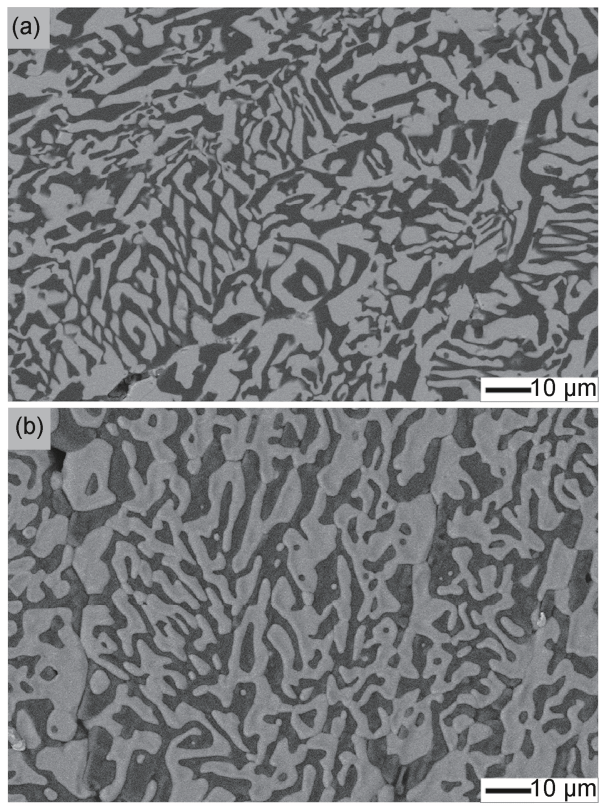

图 $5 \mathrm{Al}_{2} \mathrm{O}_{3} / \mathrm{YAG}$ 共晶陶瓷的非规则共晶形貌. (a) 抛光表面; (b) 在 空气气氛下经 $1500^{\circ} \mathrm{C}$ 处理 $20 \mathrm{~h}$ 后的表面

Figure 5 Typical microstructures of the $\mathrm{Al}_{2} \mathrm{O}_{3} / \mathrm{YAG}$ eutectics. (a) The cross-section; (b) the etched surface at $1500^{\circ} \mathrm{C}$ for $20 \mathrm{~h}$

长过冷度以满足小平面-小平面的成核与生长. 在 $\mathrm{Al}_{2} \mathrm{O}_{3} / \mathrm{YAG}$ 共晶凝固过程中, 其中一相晶体在析出时 会不断将另一相的原子排向周围的液相中, 使液相 浓度发生改变, 促进另一相在第一相周围析出, 两相 互交织缠绕耦合生长, 界面紧密相连, 形成致密的三 维共晶网络结构 (图 5), 该结构又被称为 “象形文字 (CS, Chinese script)" 结构. 从图5(b)可观察到, 在空 气条件下 $1500^{\circ} \mathrm{C}$ 处理 $20 \mathrm{~h}$ 后的共晶陶瓷表面晶体发
生了轻微粗化，但共晶间距基本与热处理前一致. 同 时, 共晶陶瓷经过此高温处理后, 样品无失重. 由于 共晶陶瓷的相界面在凝固中原位生成, 两相原子紧 密排列，与烧结材料中的晶界不同，其相界面在高温 下不易发生扩散，能够保持相对稳定.从图 6 的 HRTEM图像中可以观察到 $\mathrm{Al}_{2} \mathrm{O}_{3} / \mathrm{YAG}$ 相界面匹配良 好.

实验测得 $\mathrm{Al}_{2} \mathrm{O}_{3} / \mathrm{YAG}$ 共晶陶瓷的维氏硬度最高 达20.52 GPa, 高于Waku等人 ${ }^{[21]}$ 的报道(15 16 GPa). 根据裂纹长度计算得到共晶陶瓷的断裂韧性为 2.64 $\mathrm{MPa} \mathrm{m}{ }^{1 / 2}$, 略高于Yang等人 ${ }^{[22]}$ 的报道(约 $2 \mathrm{MPa} \mathrm{m}^{1 / 2}$ ). 由图7(a)和(b)观察到共晶陶瓷的裂纹主要发生穿晶 断裂, 基本不沿晶界扩展, 而同组分热压烧结的样品 则发生沿晶断裂. 这是因为共晶陶瓷的两相从熔体 中析出, 其相界面在原子尺度上原位结合, 结合力较 强, 同时共晶陶瓷凝固时产生的残余应力集聚在晶 体内; 烧结陶瓷的晶界由 2 个颗粒表面经传质作用形 成, 结合力较弱; 裂纹会沿结合力弱的路径扩展, 于 是产生了上述结果.

图8是在样品中观察到的接近规则共晶的特殊形 貌, (a) (d)分别为复杂规则共晶、棒状共晶、三角-圆 棒状共晶和波纹状共晶, (d) 和(e)是不同共晶形貌相 互转化的边界区域. 其中图 8(a)和(b)与 $\mathrm{Su}$ 等人 ${ }^{[2]}$ 用改 进的电子束浮区熔炼技术制得的形貌类似，而在其 他 $\mathrm{Al}_{2} \mathrm{O}_{3} / \mathrm{YAG}$ 共晶陶瓷研究中未见报道. $\mathrm{Su}$ 等人 ${ }^{[2]}$ 认 为这种现象主要由凝固时快速的晶体生长速率和小 平面相的分枝引起, 并与一些小平面-非小平面共晶 体系形貌相似，表明 $\mathrm{Al}_{2} \mathrm{O}_{3} / \mathrm{YAG}$ 体系存在不规则共

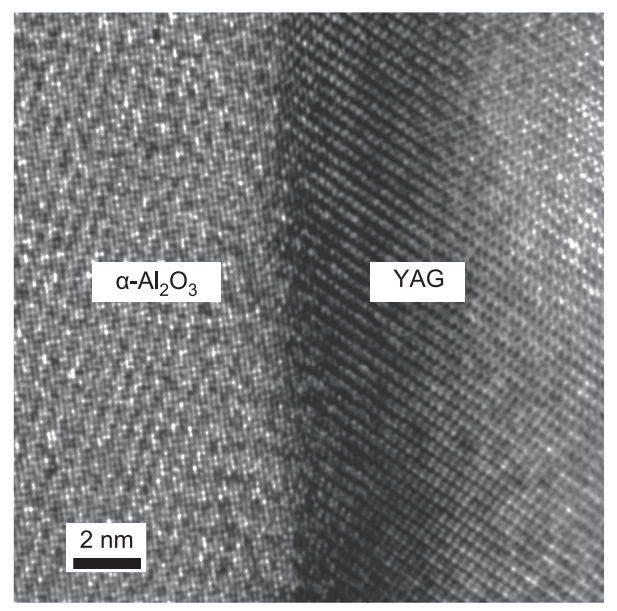

图 $6 \mathrm{Al}_{2} \mathrm{O}_{3} / \mathrm{YAG}$ 共晶陶瓷的相界面

Figure 6 Typical grain boundary between $\mathrm{Al}_{2} \mathrm{O}_{3}$ and $\mathrm{YAG}$ phases 


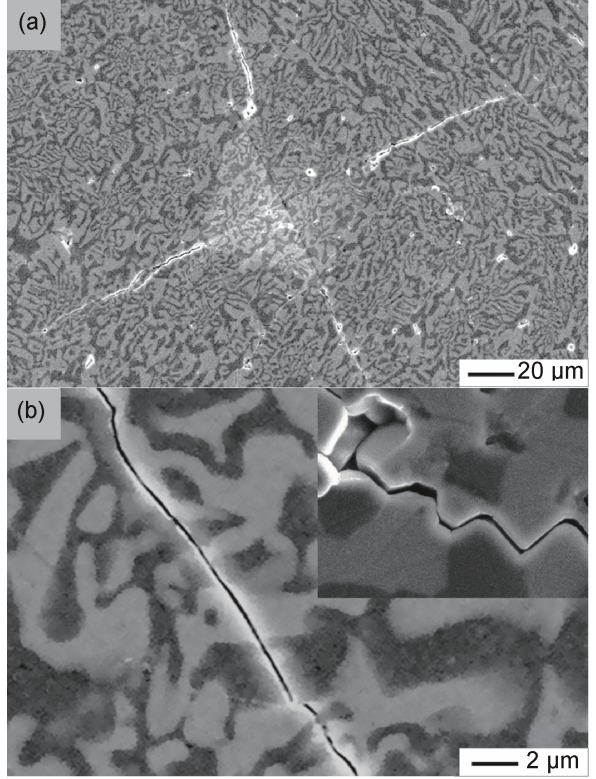

图 7 测量维氏硬度时 $\mathrm{Al}_{2} \mathrm{O}_{3} / \mathrm{YAG}$ 共晶陶瓷的表面压痕(a)与裂纹(b). 内插图为同组分热压烧结 $\mathrm{Al}_{2} \mathrm{O}_{3} / \mathrm{YAG}$ 陶瓷在同样实验条件下的裂纹 Figure 7 Indentation (a) on surface applied by hardness testing machine and the crack (b) propagation across the center. The inset of (b) is the crack in hot-press sample
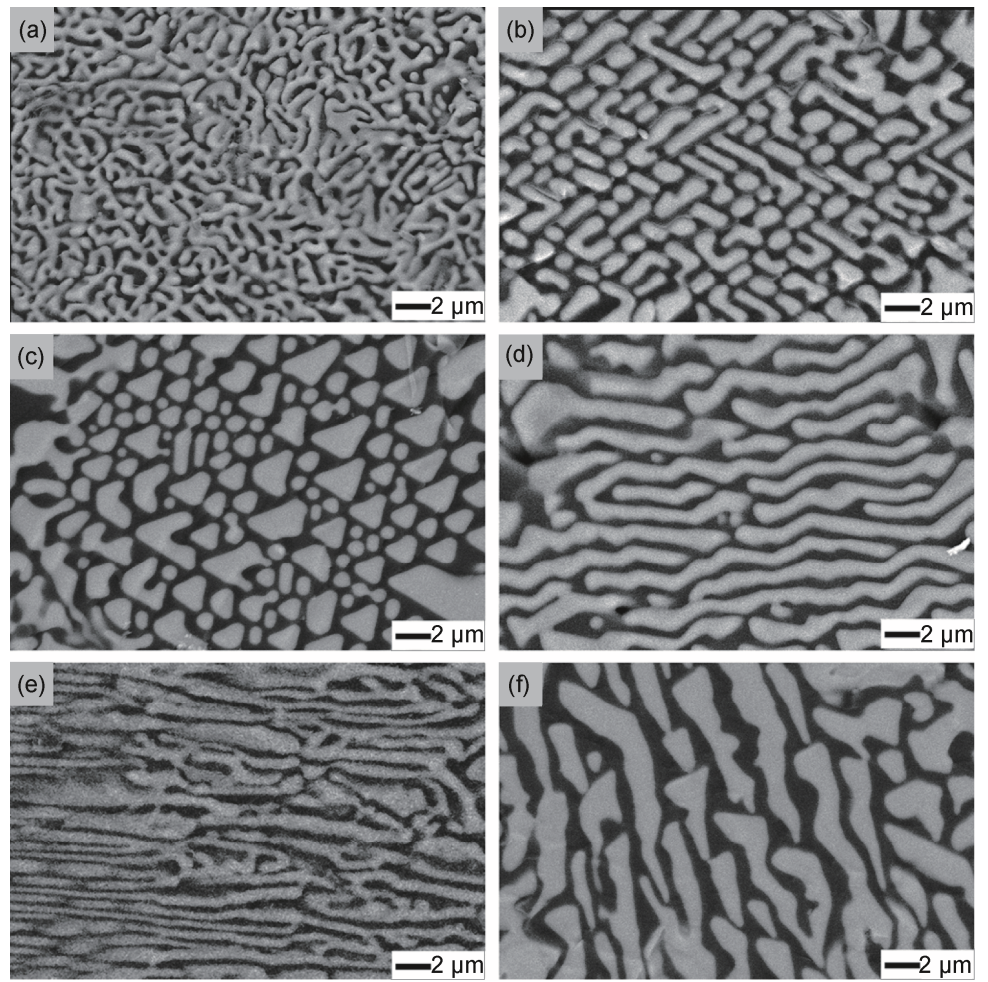

图 $8 \mathrm{Al}_{2} \mathrm{O}_{3} / \mathrm{YAG}$ 共晶陶瓷中接近规则共晶的形貌. (a) 复杂规则共晶; (b) 棒状共晶; (c) 三角-圆棒状共晶; (d) 水波纹状共晶; (e) 棒状共晶向 复杂规则共晶的转变; (f) 三角-圆棒状共晶向水波纹状共晶的转变

Figure 8 Regular eutectic growth morphologies observed in the $\mathrm{Al}_{2} \mathrm{O}_{3} / \mathrm{YAG}$ eutectic. (a) Complex regular eutectic structure; (b) rod eutectic structure; (c) rod-mix-triangle eutectic structure; (d) regular watermarked-like eutectic structure; (e) the transition of rod to complex-regular; (f) the transition of rod-mix-triangle to watermark-like 
局部区域凝固条件的不稳定导致了共晶生长方式 的变化, 得到了特殊的共晶形貌. 通过选用不同的
燃烧反应体系和温度条件，预期此方法可以应用 于其他共晶体系的制备.

\section{参考文献}

1 Waku Y. A new ceramic eutectic composite with high strength at $1873 \mathrm{~K}$. Adv Mater, 1998, 10: 615-617

$2 \mathrm{Su} \mathrm{H} \mathrm{J}$, Zhang J, Deng Y F, et al. A modified preparation technique and characterization of directionally solidified $\mathrm{Al}_{2} \mathrm{O}_{3} / \mathrm{Y}_{3} \mathrm{Al}_{5} \mathrm{O}_{12}$ eutectic in situ composites. Scr Mater, 2009, 60: 362-365

3 Yoshikawa A, Hasegawa K, Lee J H, et al. Phase identification of $\mathrm{Al}_{2} \mathrm{O}_{3} / \mathrm{RE}_{3} \mathrm{Al}_{5} \mathrm{O}_{12}$ and $\mathrm{Al}_{2} \mathrm{O}_{3} / \mathrm{REAlO}$ ( $\mathrm{RE}=\mathrm{Sm}-\mathrm{Lu}$, Y) eutectics. $\mathrm{J}$ Cryst Growth, 2009, 218: 67-73

4 Orera V M, Peña J I, Oliete P B, et al. Growth of eutectic ceramic structures by directional solidification methods. J Cryst Growth, 2012, 360: $99-104$

5 Waku Y, Nakagawa N, Ohtsubo H, et al. High temperature properties of unidirectionally solidified $\mathrm{Al}_{2} \mathrm{O}_{3} / \mathrm{YAG}$ composites. J Jpn Inst Met, 1995, 59: 71-78

6 Mizutani Y, Yasuda H, Ohnaka I, et al. Coupled growth of unidirectionally solidified $\mathrm{Al}_{2} \mathrm{O}_{3}$-YAG eutectic ceramics. J Cryst Growth, 2002, 244: 384-392

7 Waku Y, Nakagawa N, Wakamoto T, et al. The creep and thermal stability characteristics of a unidirectionally solidified $\mathrm{Al}_{2} \mathrm{O}_{3} / \mathrm{YAG} \mathrm{G}$ eutectic composite. J Mater Sci, 1998, 33: 4943-4951

8 Waku Y, Nakagawa N, Wakamoto T, et al. A ductile ceramic eutectic composite with high strength at 1873 K. Nature, 1997, 389: 49-52

$9 \mathrm{Su} \mathrm{H} \mathrm{J}$, Zhang J, Liu L, et al. Directionally solidited $\mathrm{Al}_{2} \mathrm{O}_{3} / \mathrm{Y}_{3} \mathrm{Al}_{5} \mathrm{O}_{12}$ (YAG) eutectic ceramics in-situ composites by laser remelting (in Chinese). Mater Sci Technol, 2007, 15: 741-745 [苏海军, 张军, 刘林, 等. 激光区熔定向凝固 $\mathrm{Al}_{2} \mathrm{O}_{3} / \mathrm{Y}_{3} \mathrm{Al}_{5} \mathrm{O}_{12}(\mathrm{YAG})$ 共晶的组织与断 裂韧性. 材料科学与工艺, 2007, 15: 741-745]

$10 \mathrm{Su} \mathrm{H} \mathrm{J,} \mathrm{Zhang} \mathrm{J,} \mathrm{Ma} \mathrm{W,} \mathrm{et} \mathrm{al.} \mathrm{In} \mathrm{situ} \mathrm{fabrication} \mathrm{of} \mathrm{highly-dense} \mathrm{Al}_{2} \mathrm{O}_{3} / \mathrm{YAG}$ nanoeutectic composite ceramics by a modified laser surface processing. J Eur Ceram Soc, 2014, 34: 739-744

11 Su H J, Zhang J, Ren Q, et al. Laser zone remelting of $\mathrm{Al}_{2} \mathrm{O}_{3} / \mathrm{Er}_{3} \mathrm{Al}_{5} \mathrm{O}_{12}$ bulk oxide in situ composite thermal emission ceramics: Influence of rapid solidification. Mater Res Bull, 2013, 48: 544-550

$12 \mathrm{Fu} \mathrm{X}$, Chen G, Zu Y, et al. Microstructure refinement of melt-grown $\mathrm{Al}_{2} \mathrm{O}_{3} / \mathrm{YAG} / \mathrm{ZrO}_{2}$ eutectic composite by a new method: Melt superheating treatment. Scr Mater, 2013, 68: 731-734

13 Zhao Z, Zhang L, Song Y, et al. $\mathrm{Al}_{2} \mathrm{O}_{3}-\mathrm{ZrO}_{2}\left(\mathrm{Y}_{2} \mathrm{O}_{3}\right)$ eutectic ceramics preparing by self-pressing assisting combustion synthesis under high gravity. Rare Metal Mat Eng, 2011, 40: 203-206

14 Liu G, Wang Q, Li J. Preparation of $\mathrm{Al}_{2} \mathrm{O}_{3}-\mathrm{ZrO}_{2}-\mathrm{SiO}_{2}$ ceramic composites by high-gravity combustion synthesis. Int J Refract Met Hard Mat, 2013, 41: 622-626

15 Meng F C, Fu Z Y, Zhang J Y, et al. Rapid densification of nano-grained alumina by high temperature and pressure with a very high heating rate. J Am Ceram Soc, 2007, 90: 1262-1264

16 Fu Z Y, Huang L, Zhang J, et al. Ultra-fast densification of CNTs reinforced alumina based on combustion reaction and quick pressing. Sci China Tech Sci, 2012, 55: 484-489

17 Anstis G R, Chantikul P, Lawn B R, et al. A critical evaluation of indentation techniques for measuring fracture toughness: I, direct crack measurements. J Am Ceram Soc, 1981, 64: 533-538

18 Chantikul P, Anstis G R, Lawn B R, et al. A critical evaluation of indentation techniques for measuring fracture toughness: II, strength method. J Am Ceram Soc, 1981, 64: 539-543

19 Jackson K A, Hunt J D. Binary eutectic solidification. Trans Am Inst Min Metall Pet Eng, 1966, 236: 843-852

20 Chernov A. Stability of faceted shapes. J Cryst Growth, 1974, 24: 11-31

21 Waku Y, Nakagawa N, Ohtsubo H, et al. Fracture and deformation behaviour of melt growth composites at very high temperatures. J Mater Sci, 2001, 36: 1585-1594

22 Yang J M, Jeng S M, Chang S. Fracture behavior of directionally solidified $\mathrm{Y}_{3} \mathrm{Al}_{5} \mathrm{O}_{12} / \mathrm{Al}_{2} \mathrm{O}_{3}$ eutectic fiber. J Am Ceram Soc, 1996, 79: 1218-1222

23 Minford W J, Bradt R C, Stubican V S. Crystallography and microstructure of directionally solidified oxide eutectics. J Am Ceram Soc, $1979,62: 154-157$ 


\title{
Fabrication of $\mathrm{Al}_{2} \mathrm{O}_{3} / \mathrm{YAG}$ eutectic ceramics utilizing a fast combustion reaction heating method
}

\author{
LI Bin ${ }^{1,2}$, XIANG Ming Yu ${ }^{1}$, BAO Han ${ }^{1}$, ZHANG JinYong ${ }^{1}$, WANG WeiMin ${ }^{1} \&$ FU Zheng Yi $^{1}$ \\ ${ }^{1}$ State Key Laboratory of Advanced Technology for Materials Synthesis and Processing, Wuhan University of Technology, Wuhan 430070, China; \\ ${ }^{2}$ School of Natural Science, Sun Moon University, Chungnam 336-708, South Korea
}

\begin{abstract}
$\mathrm{Al}_{2} \mathrm{O}_{3} / \mathrm{Y}_{3} \mathrm{Al}_{5} \mathrm{O}_{12}(\mathrm{YAG})$ eutectic ceramics are considered to be candidate materials for use in high temperature and oxidizing environments owing to their remarkable thermodynamic stabilities. Many techniques have been used to prepare eutectic ceramics, including the Bridgeman, edge-defined film-fed growth, micro-pulling-down, floating zone, and laser zone re-melting methods. However, these methods use complex high temperature equipment. In this work, simple equipment was used to achieve combustion reaction heating with the advantages of a high heating rate $\left(2000^{\circ} \mathrm{C} / \mathrm{min}\right)$, high temperature, and high cooling rate. Using this set-up we could melt and fabricate eutectic ceramics. The microstructure and physical properties of the obtained $\mathrm{Al}_{2} \mathrm{O}_{3} / \mathrm{YAG}$ eutectic ceramics were studied. The eutectic ceramics consisted of $\mathrm{Al}_{2} \mathrm{O}_{3}$ and YAG phases. The two phases exhibited coupled growth with well-matched and clean phase boundaries. Several special morphologies were obtained besides the typical "Chinese script" microstructure, and their formation mechanisms are discussed. The Vickers hardness of the eutectic ceramics was $20.52 \mathrm{GPa}$, which is higher than that previously reported for any binary eutectic ceramic system. The fracture toughness was $2.64 \mathrm{MPa} \mathrm{m}{ }^{1 / 2}$, which is slightly higher than about $2 \mathrm{MPa} \mathrm{m}{ }^{1 / 2}$ previously reported. The crack propagated in a straight line from the indentation corner and did not deflect at the interface between the $\mathrm{Al}_{2} \mathrm{O}_{3}$ and YAG domains, compared with the intergranular fracture in the hot-pressed sample. This weak interaction of the crack path with the microstructure was induced by the absence of residual stress and the excellent bonding between the eutectic phases. Samples treated at $1500^{\circ} \mathrm{C}$ for $20 \mathrm{~h}$ exhibited no weight loss or obvious change in microstructure, indicating the high temperature stability of these eutectic ceramics.
\end{abstract}

combustion reaction, fast heating, eutectic ceramics, $\mathrm{Al}_{2} \mathrm{O}_{3} / \mathrm{YAG}$, eutectic microstructure

doi: 10.1360/N972014-00450

·科学新闻 .

\section{稀土顺丁橡胶科研成果实现工业化应用}

北京化工大学吴一弦教授研究组致力于稀土催化剂、 丁二烯可控配位聚合、聚合物结构与性能等研究, 取得一系 列研究成果与创新技术. 基于该研究成果与创新技术, 由 中国石化北京燕山分公司与北京化工大学等共同开发了 3 万吨/年稀土顺丁橡胶工业成套技术，建成了中国石化第一 套稀土顺丁橡胶的工业化生产装置, 一次成功打通了装置 全流程，生产的高性能稀土顺丁橡胶产品(牌号：BRNd40, BRNd50, BRNd60)的加工及应用性能与国际同类产品相当, 为生产绿色轮胎提供了高性能合成橡胶基础材料. 中国石 油化工集团公司组织的专家鉴定委员会一致认为该项目所 开发的 3 万吨/年稀土顺丁橡胶工业成套技术具有创新性和 自主知识产权，整体技术达到了国际先进水平。在 2014年 12月 10 日召开的中国石化“十条龙”科技攻关工作会议上，3 万吨/年稀土顺丁橡胶工业成套技术项目顺利“出龙”.

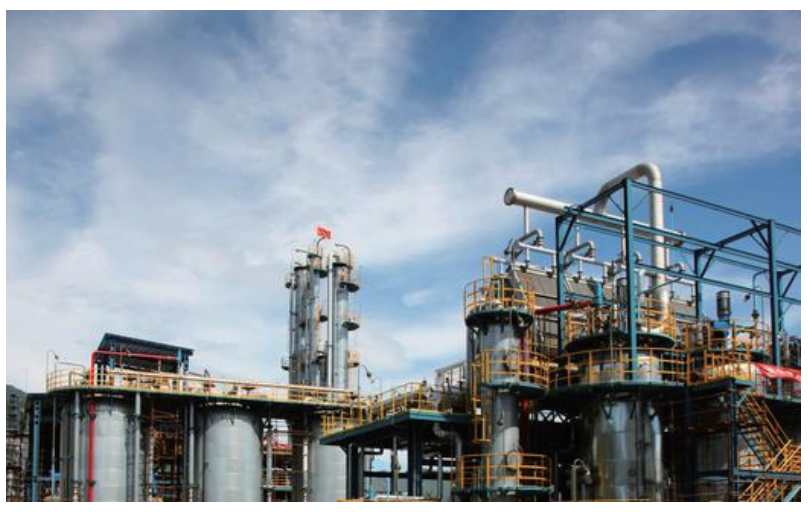

该项目是吴一弦教授研究组多年来坚持产学研用相结 合, 将基础研究创新成果与工业生产紧密结合的又一硕果. 